\title{
La Bibliotecología y Documentación en su cruce con otras disciplinas. Su importancia para la organización del conocimiento
}

\author{
Library and Information Science at its crossroads with other \\ disciplines. Its importance for the knowledge organization
}

\author{
José Antonio Moreiro-González \\ Universidad Carlos III de Madrid, Madrid, España \\ Contacto: jamore@bib.uc3m.es \\ https://orcid.org/0000-0002-8827-158X
}

\begin{abstract}
Resumen
Reflexión en torno a la conformación disciplinaria de la Bibliotecología y Documentación. A partir de consideraciones válidas para la generalidad de las especialidades se atiende a los conceptos de multidisciplinariedad, interdisciplinariedad y transdisciplinariedad, para descender desde ellas a la conformación y evolución de dicha especialidad y justificar la configuración de sus paradigmas teóricos, sin olvidarnos de sus aplicaciones prácticas e incluso de las características de su enseñanza actual. El repaso conceptual se fija en los grandes momentos del desarrollo evolutivo para identificar las intervenciones de carácter multidisciplinario e interdisciplinario. En especial se atiende al extenso periodo de la bibliografía, así como al momento de conformación interdisciplinario de la documentación, primero, y luego de la Ciencia de la Información. Se llega finalmente a considerar la progresión transdisciplinaria actual en el ejercicio profesional y en la formación superior de nuestro sector.
\end{abstract}

Palabras claves: Bibliotecología y Documentación; Multidisciplinariedad; Interdisciplinariedad; Transdisciplinariedad; Proceso evolutivo; SOC

\begin{abstract}
Is addressed to the disciplinary conformation of Information science. The concepts of multidisciplinary, interdisciplinary and transdisciplinary are fixed in relation to all scientific, technical and humanistic specialties. To drop from them to the Information science and reasoning the compositional baggage of its conceptual aspects and their practical applications and even teaching. The review focuses on the great moments of the evolutionary process to analyze the multidisciplinary and interdisciplinary interventions. Particularly during the long bibliographic period. Also on the formation of interdisciplinary Science of documentation, first, and the Information science, after. At length, the progress of transdisciplinary on performance and current university education is attended to.
\end{abstract}

Keywords: Library and Information Science; Multidisciplinary; Interdisciplinary; Transdisciplinary; Evolutionary process; KOS 


\section{Introducción}

La revolución científica racionalista organizó el conocimiento en disciplinas con el fin de acotar el espacio de actuación de cada una de ellas, así como de ordenarlo de acuerdo con la necesaria eficacia para planear y sistematizar su adiestramiento y doctrina, su indagación o experimentación e, igualmente, las correspondientes destrezas y prácticas profesionales. De este modo, cada disciplina pasó a tener una percepción característica derivada de la semántica de mundo que consideraba propia, siempre con referencia a un contexto que precisaba su objeto de estudio y que se constituía y ejercía a partir de un entorno conceptual concreto, de unos métodos de investigación específicos y de una praxis, un trabajo y unos procedimientos que se creían, hasta cierto punto, privativos. A partir de esa herencia se hace evidente, de forma inmediata y manifiesta, el interés y la actualidad que muestra, para quienes se relacionan con la Bibliotecología y Documentación, el hecho de abordar su composición y evolución disciplinarias. Esto a partir del largo recorrido realizado hasta llegar a un presente de confluencia y cambio de continuo renovado y hasta de frecuente desorientación. Para quienes estudian, investigan, enseñan y trabajan en dicho sector tiene una importancia fundamental conocer su naturaleza y la situación que ocupa en el mundo científico y académico. Aunque sea un referente continuo y una explicación repetida, se trata de considerar la participación de otros sectores del conocimiento en la fundamentación teórica y en la colaboración práctica a la hora de desarrollar proyectos y aplicaciones conjuntos. Al tiempo que se entienden las grandes líneas seguidas desde las ideas y aplicaciones iniciales hasta alcanzar a explicarse el actual estado de las cosas y las tendencias en su contexto, centrado en especial respecto a los sistemas de organización del conocimiento (SOC).

Se atiende, pues, a un asunto que ofrece un indudable estímulo para comprender la conformación de los conceptos y de las aplicaciones en Bibliotecología y Documentación. La elaboración de esta reflexión se ha derivado de la identificación y valoración inicial de las fuentes pertinentes, de cuyos contenidos se han deducido, de forma esquemática, la evolución histórica de nuestra actividad y las bases disciplinarias sobre las que emergieron. Siguiendo su consolidación y su presencia en las características que podemos considerar más permanentes, hasta identificar las que determinan la actualidad. Los contenidos 
de esos recursos se han seleccionado y valorado para contrastar sus aportaciones con idea de que sirviese como base argumental para esta breve reflexión.

\section{La relación entre disciplinas diferentes}

La multidisciplinariedad, la interdisciplinariedad y la transdisciplinariedad se reflejan en los aspectos teórico-conceptuales, en los procesos de investigación, en las ejecuciones de proyectos y en las acciones aplicadas. Desde la revolución científica positivista, el conocimiento se organizó por disciplinas, pero ninguna de ellas estaba totalmente cerrada. Sus límites mantuvieron siempre un contacto permanente con otras disciplinas en el que se abrían espacios de intersección. No obstante, los investigadores y los métodos seguidos por cada disciplina mostraban una línea de actuación conforme a su objeto de estudio:

- marcos teóricos,

- métodos de investigación,

- $\quad$ prácticas y técnicas propias.

Mientras que por multidisciplinariedad se entiende la colaboración entre especialidades para abordar un asunto en común, de forma que las diversas áreas se coordinen para poder cooperar, sin buscar integrarse, con la finalidad de completar proyectos o servicios, integrando aplicaciones con los resultados de la investigación. En esta colaboración, cada especialidad trabaja autónomamente, con sus propios estándares y procedimientos, en una aproximación yuxtapuesta de intereses dentro de un entorno en el que se hace posible la colaboración.

En la interdisciplinariedad varias áreas aportan sus conceptos, métodos y práctica al estudio común de un objeto que es distinto a los objetos de estudio propios de cada una de ellas. Las relaciones interdisciplinarias se producen, igual que en el caso anterior, en un espacio concreto de colaboración. Pero en este caso el ámbito determina nuevas competencias y prácticas por la cuales los conceptos y valores de las disciplinas concurrentes se tienen que combinar e integrar. Desde la aplicación práctica se hace evidente la necesidad de que las disciplinas cooperen. La interdisciplinariedad presenta una mayor exigencia que la multidisciplinariedad, ya que en esta los objetos de análisis se sitúan, en gran parte, limitados dentro de una disciplina, mientras que en la interdisciplinariedad los objetos de análisis se 
perciben como contribución hacia un nuevo conocimiento. De forma que, desde el intercambio disciplinario, se consiguen beneficios recíprocos, al tiempo que se fomentan transformaciones aplicativas y metodológicas, pero además teóricas y docentes (Newell, 2001).

Finalmente, la propuesta transdisciplinaria consiste en conseguir eficacia dentro de la condición vinculada y de coprotagonismo en la que se desarrolla la actividad científica, técnica y humanística en la actualidad. La vigente realidad en red no puede analizarse sin emplear una visión amplia que permita encontrar un nuevo sistema para interpretar su complejidad (Martínez, 2003). Se vuelve así necesario el estudio de sus rasgos a partir de un tipo coherente de niveles y objetivos múltiples que cambian según sea el asunto principal que justifica la actuación de cada uno de los sectores que intervienen en su tratamiento, pero también de acuerdo con las circunstancias. En definitiva, se rebasan los límites habituales de esas disciplinas para apreciar los espacios de colaboración. De esta forma, se avanza hacia una consideración teórica del conocimiento, establecida desde la mutualidad de los conocimientos.

La transdisciplinariedad aborda el conocimiento y la práctica con una perspectiva integral. Los hechos de nuestro mundo son complejos y ofrecen muchas facetas para abordar su conocimiento. No se pueden comprender cuando su estudio o tratamiento se parcializa. Así lo reconocía Morin (1990), pues si las disciplinas limitan los planteamientos, los conocimientos se compartimentan, lo que dificulta entenderlos en su contexto, al olvidarse de las relaciones y la coherencia de conjunto. Si optamos por parcializar su tratamiento, entonces perjudicamos su comprensión. Nos movemos en un escenario de visiones acumuladas y desiguales imposibles de cumplir desde un planteamiento disciplinario. Así lo imponen una sociedad y una vida cuyas cuestiones se abordan desde la conexión, la cooperación y la fugacidad. Cualquier acción exige salirse del encasillamiento al que obliga una única especialidad y abrirse a todas las áreas del conocimiento que puedan intervenir en su análisis e investigación, que serían inabordables por su condición compleja que exige tratamientos compuestos y que, además, se debe enfocar siempre con la intención de producir nuevo conocimiento (Velilla, 2002). En estas circunstancias de naturaleza compleja los límites de las disciplinas concretas se 
desdibujan. Aquí subyace la causa de que, por el mayor dinamismo de cualquier planteamiento transdisciplinario, la mayor parte de sus aplicaciones se han efectuado en un campo de tanta complejidad como el de la educación (Bolaños, Bueno y Alves, 2013). Se abandona, de esta manera, el camino de fraccionar y clasificar; en su lugar se busca ahora armonizar, acoplar y acomodar las ideas para procurar que las diferentes disciplinas interesadas acaben por encajarse desde sus principios comunes en un mismo procedimiento y método de investigación, sin que ninguna de ellas predomine sobre las demás.

No hay que pensar que la multidisciplinariedad, la interdisciplinariedad y la transdisciplinariedad sean planteamientos excluyentes, si no que, además de haber tenido una aparición gradual, se necesitan y son nociones que suelen funcionar juntas. Es así porque se permiten asociar tanto con relación a los aspectos propios de la concepción teórica como a la hora de ejecutar un proyecto investigador o de realizar cualquier acción complicada. La proximidad entre la multidisciplinariedad y la interdisciplinariedad resulta evidente, pues sin aquella no hay posibilidad de que esta se cumpla. Resulta menos evidente la integración con la transdisciplinariedad, ya que, sin abandonar el provecho que causan sus aportaciones, supera a ambas tanto en los fines que persigue como en las propuestas que realiza, pues se acerca a la vida desde sus heterogéneas facetas y categorías (Pérez y Setién, 2008). La interdisciplinariedad y la transdisciplinariedad también coinciden en que ambas se proponen cooperar en relación con unos contenidos, estructuras y áreas de contacto de unas disciplinas en variación constante. Un paso más allá lo da la transdisciplinariedad que reivindica modificaciones en la naturaleza de los conceptos y de la metodología para alcanzar nuevas conceptualizaciones y resultados a partir de elementos de distinta naturaleza.

La Bibliotecología y Documentación siempre ha contribuido a la comunicación científica en interactuación con todas las disciplinas científicas y humanas. Por ello, siempre tuvieron vocación interdisciplinaria. Su actuación siempre se enriqueció con diferentes conocimientos — rasgo multidisciplinary manifestó reciprocidad epistemológica y metodológica, característica interdisciplinaria también. Ahora han permeabilizado sus límites disciplinarios y se han abierto hacia una teoría y práctica transdisciplinarias. 


\section{Jalones de la interdisciplinariedad en Bibliotecología y Documentación}

Desde el siglo V a. C., y hasta el siglo XVII d. C, se siguió la misma estrategia en nuestra área: optar por una contribución multidisciplinaria para establecer los fundamentos conceptuales; mientras que, a la hora de abordar los problemas de transmisión, conservación y acceso a la información, se empleaban soluciones interdisciplinarias. A ello obligaba el hecho de que la Bibliotecología y Documentación siempre actuó como disciplina auxiliar de todas las demás, ya que su función se ha cumplido siempre sobre cualquier tipo de conocimiento grabado. Ello desde una base multidisciplinaria, pues desde sus orígenes se conformó con aportaciones de varias disciplinas, mostró siempre una actuación interdisciplinaria y ella misma acabó formando parte de la aplicación de otras disciplinas. Como antecedentes remotos de la actuación documental contamos con varias acciones emprendidas durante el clasicismo heleno. El ejemplo emblemático, que no único, es la organización de la Biblioteca de Alejandría, arquetipo sin duda de la conjunción inicial de los conceptos multidisciplinarios y de la aplicación, que comenzaba a hacerse interdisciplinaria a los fondos allí custodiados. Llama la atención que la denominación de esos fondos fuese la de Museion, en definitiva, una agrupación de objetos de información, como hoy prefieren las normas Z39.19 (2005) y BSI Group (2005-2007), por encima de las diferentes unidades de información que contenía, pues a la par era biblioteca (rollos), archivo (documentos originados en la gestión pública) y museo. Allí se establecieron los precedentes de las técnicas y de los procesamientos de la información seguidos hasta el presente y que, además, permitió a sus colecciones disponer de los sistemas de catalogación y clasificación originarios:

- Pinakes: división del fondo de la biblioteca en 127 especialidades conforme con lo expuesto por Aristóteles (1995) en la Teoría de las taxonomías, que fijó en su Lógica. Se aplicó a las técnicas de control de las obras de todos los campos disciplinarios. Se formó un repertorio que permitía buscar la información por especialidades y en el que los rollos aparecían catalogados y resumidos a partir de una clasificación acorde con su mensaje. 
- Syllabus: basado en los principios metafísicos de la identificación y del establecimiento de la diferencia entre los conceptos, era una técnica de descripción que se estableció como el antecedente indudable de nuestros registros bibliográficos, al mismo tiempo elementos distribuidores de materias, auténticos instrumentos de búsqueda bibliográfica.

- Canon: de origen filológico, estableció los principios para la teoría y práctica de los repertorios secundarios. Ofrecía listados críticos de los autores y de sus títulos para favorecer el acceso a los textos existentes y ayudar a comprenderlos. Se la ha considerado "Nómina de los escritores que podrían considerarse como modelos en cada uno de los géneros literarios" (Millares, 1971).

Tabla 1. Multidisciplinariedad en la solución de los problemas de acumulación informativa

\begin{tabular}{|l|l|l|l|}
\hline \multicolumn{1}{|c|}{ TÉCNICAS } & \multicolumn{1}{|c|}{ Pinakes } & \multicolumn{1}{c|}{ Syllabus } & \multicolumn{1}{c|}{ Canon } \\
\hline ORIGEN & $\begin{array}{l}\text { Teoría de las } \\
\text { taxonomías-lógica } \\
\text { aristotélica }\end{array}$ & Metafísica & Filología \\
\hline NATURALEZA & $\begin{array}{l}\text { Repertorio que } \\
\text { clasificaba los } \\
\text { fondos en 127 } \\
\text { especialidades }\end{array}$ & $\begin{array}{l}\text { Descripción de la } \\
\text { identificación y } \\
\text { establecimiento de } \\
\text { la diferencia entre } \\
\text { los conceptos }\end{array}$ & $\begin{array}{l}\text { Teoría de los } \\
\text { repertorios }\end{array}$ \\
\hline secundarios \\
\hline TÉCNICAS & $\begin{array}{l}\text { Control de las obras } \\
\text { de cualquier campo } \\
\text { del saber: catalogar } \\
\text { y condensar los } \\
\text { documentos por su } \\
\text { mensaje }\end{array}$ & $\begin{array}{l}\text { Registros de } \\
\text { identificación y } \\
\text { elementos } \\
\text { distribuidores de } \\
\text { materias, } \\
\text { instrumentos de } \\
\text { búsqueda } \\
\text { bibliográfica }\end{array}$ & $\begin{array}{l}\text { Listados críticos } \\
\text { de los } \\
\text { autores-modelo y de } \\
\text { sus títulos, para dar } \\
\text { acceso a los textos } \\
\text { existentes y ayudar a } \\
\text { su comprensión }\end{array}$ \\
\hline
\end{tabular}

Fuente: Elaboración propia 
De forma que la epistemología original tuvo ya carácter multidisciplinario, el cual se mantuvo en las siguientes grandes corrientes conceptualizadoras: la bibliografía, la bibliología y la documentación. La actuación técnica hacia la solución de los problemas se hizo también a partir de conceptos generados en otras disciplinas. Así, sus ideas se transformaron en métodos para procesar la información.

La poca presencia del libro en la Edad Media parece explicarse, según Ortega (1984), desde su utilización infrasocial, por lo que, al limitarse la posibilidad de consulta a las bibliotecas monásticas, bastaba con la identificación bibliográfica. Este hecho cambió con la llegada de la imprenta, que permitió obtener con facilidad muchas copias idénticas de cada original. La abundancia de ejemplares, junto al afán cultural humanístico, impulsó los estudios bibliográficos a lo largo del siglo XVI para informar sobre las existencias mediante la aplicación de normativas para la descripción técnica.

Tabla 2. Multidisciplinariedad en la elaboración de las bibliografías

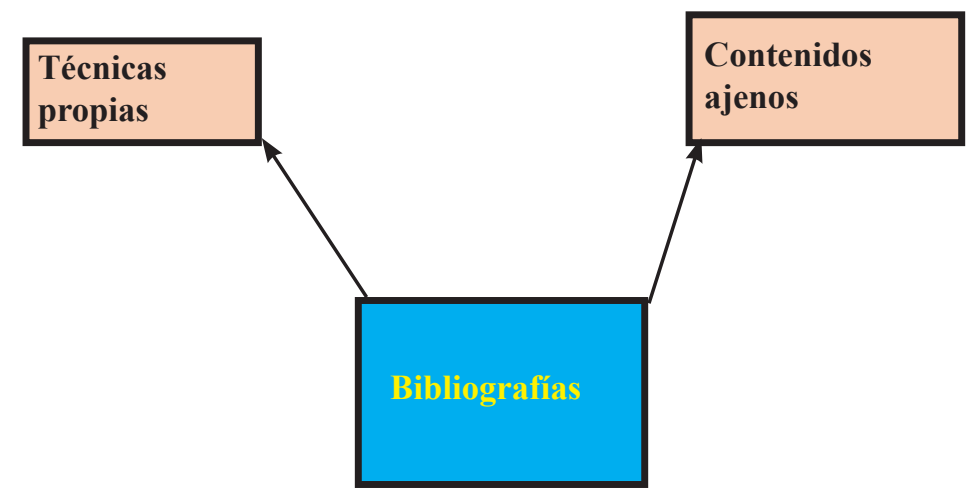

Fuente: Elaboración propia

En el siglo XVII el libro dejó de ser el único tipo de impreso, al aparecer las revistas para cubrir las necesidades informativas de los científicos. Se daba un paso más hacia el surgimiento del periodismo científico. Las publicaciones periódicas llegaron como necesidad de un nuevo vehículo para comunicar las aportaciones científicas. Se necesitaba un medio más diligente que la edición de libros y más sistemático que las reuniones de las sociedades científicas. Las publicaciones 
periódicas se volvieron el recurso más adecuado para trasladar los conocimientos científicos, porque desde el primer momento contenían no solo información original destinada a acopiar trabajos científicos breves, sino también referencial que permitía el acceso a los documentos primarios, función que luego cumplirían las revistas de resúmenes y las bases de datos. Ser a la par vehículo y depósito de información venía exigido por la comunidad científica ante el gran incremento del conocimiento que buscaba nuevos canales de transmisión.

\section{Tabla 3. Multidisciplinariedad con la aparición de las publicaciones} periódicas

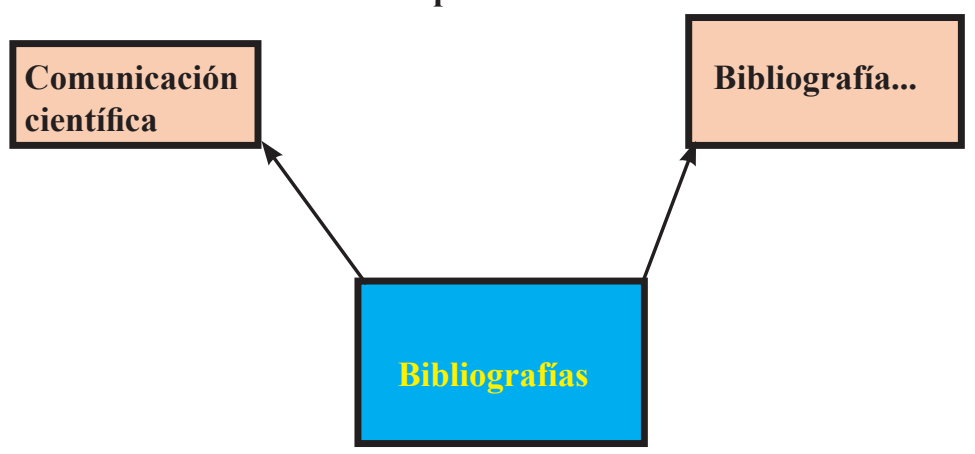

Fuente: Elaboración propia

\section{Sistematización de la documentación e interdisciplinariedad}

Con la transformación social, tras el triunfo de la Revolución francesa, el libro pasó a ser fundamento de todo lo social. A ello se añadió el crecimiento cognitivo impulsado por la ciencia positivista. Como reflejo, se dispararon el número y tamaño de las ediciones junto a los estudios del libro, su producción y acceso, además de la producción de nuevos soportes documentales. Desde los primeros intentos de fundamentación disciplinaria de la documentación, al comienzo del siglo $\mathrm{XX}$, los recursos semánticos se establecieron como vía por la que organizar y controlar la complicada existencia de los documentos. Destaca por la seriedad de los planteamientos y el éxito a la hora de sistematizar el almacenamiento y la recuperación de la información, los diferentes proyectos encabezados por Otlet y La Fontaine que aspiraron a ofrecer toda la información existente y fundaron el 
Instituto Internacional de Bibliografía. Sería la oficina operativa donde se crearía un gigantesco cuerpo referencial, el Repertorio Bibliográfico Universal (RBU) para poder utilizar la información registrada en todo tiempo, lugar y sobre cualquier asunto, y cuya organización facetada permitió idear un sistema clasificatorio de aplicación universal, la CDU, adaptación de la Dewey Decimal Classification. De esta manera quedaba reconocida la capacidad del mensaje documental para sistematizar la información desde categorías lingüísticas, apareciendo los primeros intentos de alcanzar lenguajes para dialogar con los documentos. La CDU fue un nuevo lenguaje en el que los números expresaban la relación de similitud, dependencia o diferencia entre los conceptos científicos (Moreno y Borgoñós, 1999). Como lenguaje codificado, que solemos agrupar con la denominación de Sistemas de Clasificación, está inspirado en las estructuras jerárquicas del pensamiento aristotélico-escolástico y, posteriormente, racionalista. Esta situación contextualiza el intento de Otlet (1934) de alcanzar un Repertorio Bibliográfico Universal, que precisaba de un lenguaje de comprensión internacional que contuviese la totalidad de materias tratadas por los documentos. Los intentos clasificadores anteriores fueron localistas, mientras la CDU supuso un acuerdo internacional y dio origen a las organizaciones lingüísticas documentales.

\section{Tabla 4. Documentación, ciencia de aplicación global}

Agrupaba las bases de la multidisciplinariedad de nuestro campo desde la antigüedad:

- Bibliotecología

- Bibliografía

- Retórica

Junto a la teoría del conocimiento, en el cruce entre las ciencias fundamentales y los modos de comunicación.

Además, en conexión interdisciplinaria con otras ciencias que se ocupan de los documentos y de la información:

- Lógica,

- Psicología,

- Tecnología,

- Sociología. 


\section{Aumento interdisciplinario en el paso de la Documentación a la Ciencia de la Información}

Se puede considerar la interdisciplinariedad de la Bibliotecología y Documentación, en su fase de Ciencia de la Información (que empleamos en este texto como sinónimo de Bibliotecología y Documentación), como una estrategia posmoderna. Mediante esta se pasa de las estructuras científicas de carácter jerárquico a las de red, como consecuencia de la intervención de los ordenadores en los procesos aplicados y de investigación. Las diferentes disciplinas ampliaron su campo de actividad hasta cruzarse con otras. Ello fue más notorio en campos como el nuestro, que siempre ocupó espacios de intersección. Precisamente, la integración de las disciplinas que habían fundamentado nuestros conceptos y aplicaciones desde la antigüedad acabó produciendo la llegada de la Ciencia de la Información en la articulación de:

- tecnologías de la información y la cibernética,

- métodos lingüísticos,

- mecanismos lógico-semánticos,

- teoría de la comunicación y de la información,

- además de los campos de origen del conocimiento tratado.

La aparición transformadora de la Ciencia de la Información surgió en un cruce interdisciplinario. La considerada primera definición de la disciplina relacionaba la Ciencia de la Información con métodos y contenidos provenientes de "las matemáticas, la lógica, la lingüística, la psicología, la tecnología de los ordenadores, la investigación operativa, las artes gráficas, la comunicación, la biblioteconomía, la gestión, y algunos otros campos"(Georgia Institute of Technology, 1962). En su formación intervinieron la Lingüística, la Teoría de la Comunicación y de la Información, la Lógica y la Cibernética (Wellisch, 1972). Vemos así cómo la Ciencia de la Información está especialmente vinculada con la Bibliotecología, con la Documentación y con la Informática. La relación con esos campos disciplinarios se hizo notar desde los primeros intentos de conceptualización. Siempre que se hacía referencia a la Bibliotecología o a la Documentación, suponía incluir las disciplinas sobre las que ellas se habían conformado, de modo que la constitución de la Ciencia de la Información los heredaba, a la par que interconectaba con campos nuevos 
para responder al reto del nuevo contexto informático, empresarial y social. De este modo lo resaltó Taylor (1966), pues frente a la preponderancia de lo aplicativo que era común hasta entonces, planteó una doble vertiente de estudio, la teórica $-\mathrm{y}$ prioritaria - del conocimiento especializado (relacionado con las Matemáticas, la Lógica, la Psicología y la Lingüística), y la operativa, referente a las tareas de recuperación, organización y difusión (consideradas desde la metodología científica, la Bibliotecología y la Informática).

Tabla 5. Propuesta interdisciplinaria de Taylor para la Ciencia de la Información

Matemáticas

Estudio teórico

(conocimiento especializado)

Aplicación a la recuperación, organización y difusión

\section{Lógica}

Psicología

Lingüística

Metodología científica

Biblioteconomía

Informática

Fuente: Elaboración propia

En la representación de la información, la situación cambió cuando hubo que desarrollar las indizaciones coordinadas para recuperar la información. La operatividad característica de los ordenadores llevó a los sistemas de clasificación a un segundo plano, lo cual limitó su uso a la organización de las colecciones bibliotecarias. Ahora, las técnicas aplicadas perseguían la automatización documental del análisis del contenido de la información integrada en bases de datos, cuya recuperación requería utilizar lenguajes naturales controlados. La recuperación se decantaba por indizaciones coordinadas sobre términos extraídos del lenguaje natural y combinados mediante operaciones de lógica matemática, en una conceptualización y aplicación bien distintas de las que la Documentación había seguido hasta entonces. 
Tabla 6. Interdisciplinariedad en Ciencia de la Información

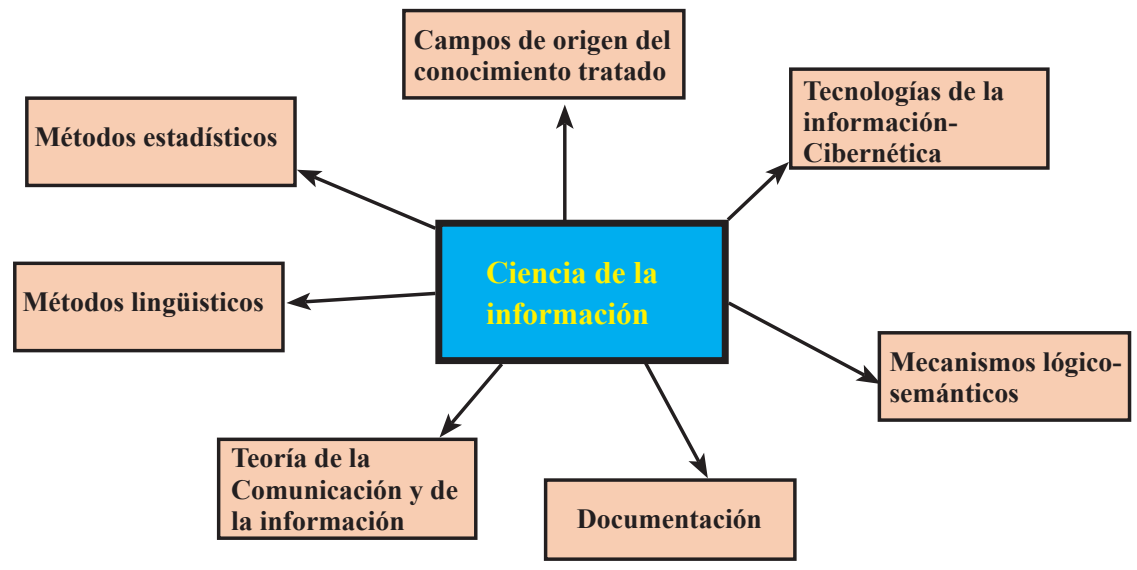

Fuente: Elaboración propia

Este hecho se convirtió en uno de los fundamentos de la Era de la Ciencia de la Información, tal como lo reconoció Saracevic (1979). Se veía posible, por primera vez desde Otlet, registrar todo el conocimiento existente, incluso el no publicado, y realizar un índice universal de la ciencia gracias al desarrollo informático. Lo llevó a la práctica Mortimer Taube (1959) cuando planteó la noción y el proceso para elaborar un índice coordinado. Llamó a este método indización por unitérminos. Se trataba del primer índice no tradicional que impulsó la indización coordinada y la recuperación por ordenadores. Fue el método antecedente de los descriptores. Cada concepto dentro de un fondo se relacionaba a través de una tarjeta, que a su vez se controlaba mediante un glosario de unitérminos. Coincidió con la aparición del primer tesauro operativo. El control de la búsqueda y recuperación de la información tuvo un gran impulso en el Informe Weinberg (1963) que reconocía la necesidad de "lograr la compatibilidad de los lenguajes de indización, y de sus formatos, procesos y convenios, particularmente cuando se contempla el factor de la mecanización" (traducción propia).

El sistema de Taube ofrecía su mayor debilidad en el origen extractivo de los términos y en su representación por unidades léxicas. Para evitarlos, Mores (1959), con el Zato-coding, ideó el uso de unos términos normalizados llamados 
descriptores. De esta forma, las relaciones con la Lingüística aparecieron de inmediato y se constituyeron en una de las características básicas del concepto de Information Science. Así pues, en la década de 1960 la indización se manejaba a partir de conceptos lingüísticos. Entonces hubo que sistematizar las estructuras y normas lingüísticas para informatizarlas. La contribución lingüística a la Bibliotecología y Documentación se ha beneficiado directamente del procesamiento automático del lenguaje y ha traído gran provecho para la gestión del contenido documental. Se volvió propio entender los mecanismos lógico-semánticos inherentes a todo texto.

Los tesauros se han establecido como el símbolo de los lenguajes controlados para representar los contenidos documentales. En su elaboración y uso participan documentalistas como expertos en los sistemas de información a los que van destinados, e incluso como normalizadores de los términos, labor que pueden realizar terminólogos de una disciplina; en definitiva, especialistas del ámbito de aplicación, además de informáticos para su gestión automatizada. Su elaboración y uso son consecuencia de una contribución interdisciplinaria. Pero su gestión es muy pasiva en relación con la rapidez de respuesta que requiere el tratamiento informativo en la web (Sánchez-Cuadrado, Colmenero-Ruiz y MoreiroGonzález, 2012). Además de ser un vocabulario que conecta el pensamiento humano con los documentos $\mathrm{y}$, aunque se vale de medios automatizados, no cuenta con los ordenadores para el procesamiento automático de la información, por lo que la comprensión es siempre mental. Precisamente la complejidad subsiguiente a los procesos de entendimiento semántico con los ordenadores hará coincidir los intereses de múltiples áreas en proyectos conjuntos esclarecedores de objetivos coincidentes, pero con diferentes niveles de actuación.

\section{Redes semánticas y transdisciplinariedad}

Five Intersecting Tetrahedra (FIT) es una gráfica que capta nuestra atención de inmediato por su diseño. Se entiende como la representación simbólica de la transdisciplinariedad. Cada uno de sus elementos geométricos está determinado por cuatro caras y cada uno se enlaza con los otros, por lo que representa la complejidad. Transdisciplinariedad y complejidad están estrechamente unidas como formas de pensamiento relacional y como interpretaciones del conocimiento 
desde la perspectiva de la vida humana y del compromiso social (Freire). La perspectiva transdisciplinaria armoniza sus elementos constituyentes hasta conseguir un núcleo participado. Ello establece un espacio válido para tratar los asuntos propios de las Ciencias Sociales. Precisamente por su complejidad queremos emplearla para representar el análisis semántico.

\section{Tabla 7. Análisis semántico para la representación con la gráfica FIT}

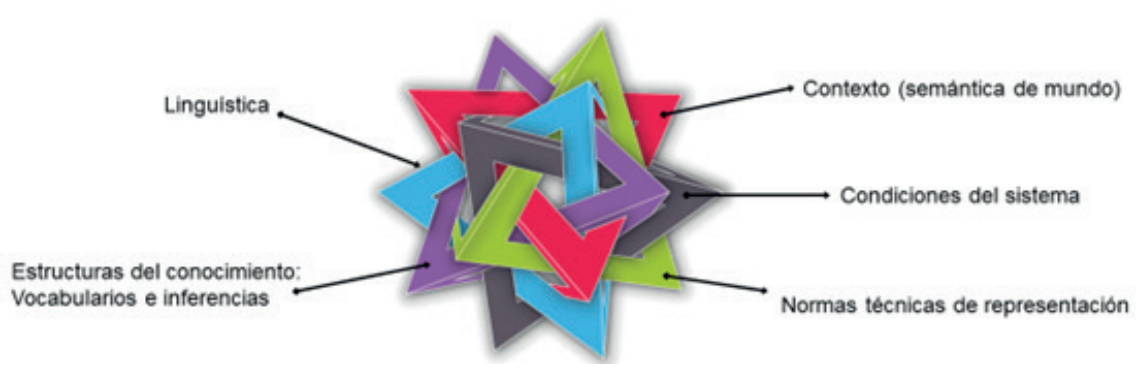

Fuente: Elaboración propia

La progresión fue rápida en las décadas finales del siglo XX y se realizó dentro de una estrategia que progresó con celeridad. Se pasó, así, desde la jerarquía estructural de los lenguajes documentales a unos vocabularios en red como respuesta a la intervención progresiva y determinante de los ordenadores, si bien fue la llegada de la web la que obligó a los lenguajes documentales a adaptarse. Si el tesauro prototípico (ISO: 2788,1986 ) se basaba en una estructura jerárquica de descriptores con relaciones de asociación, las redes semánticas ampliaron la perspectiva para responder a las necesidades de la web. Al mantener las relaciones jerárquicas terminológicas se pasó a admitir tantas asociaciones como podían manifestarse en la semántica de mundo, cuyas representaciones se mostraban con toda plasticidad mediante los Topic maps. De esta forma, hubo una gran progresión semántica incluso por vía expresiva.

Aun así, el carácter terminológico de los vocabularios controlados se ha mantenido en las nuevas normas que regulan estos cambios: Z39.19-2005, BSI Group 2005-2007 e ISO; 25964-1-2011. Tampoco cambió la organización taxonómica para estructurar los descriptores junto a la presencia de mayores relaciones, no obstante 
fue novedosa la fuerza de la interoperabilidad entre diferentes vocabularios. Esta interdisciplinariedad de aplicación de términos y de relaciones venía a permitir la reutilización de términos y esquemas. Para ello se hace necesario el descubrimiento efectivo de los conceptos por procesos automatizados y, luego, integrarlos y reutilizarlos entre diferentes aplicaciones, por tanto, con un grado de complejidad alto.

En tal sentido, para una estructuración de los datos y una visualización más fácil, se unió la intervención de redes como la teoría de los grafos para organizar los conceptos y las relaciones que se dan entre conceptos, provenientes del ámbito educativo, de la Sociología y de la Psicología. Se aprovechó eso para hacer explícitas las relaciones mediante el uso de expresiones verbales que complementaban a los tradicionales tesauros estáticos de sustantivos y expresaban las asociaciones que existen entre los conceptos empleando todas las posibilidades del lenguaje natural. Esta representación del conocimiento se lograba mediante frases simples para elaborar los grafos: sujeto - verbo - predicado, procedimiento que después adoptó el RDF como modelo de grafo para formalizar el conocimiento en las ontologías que, como redes ya semánticas, además de representar, tienen que razonar. De modo que se estableció un paralelismo necesario entre los grafos de las redes semánticas y la creación de sentencias RDF. 
Tabla 8. Disciplinas que participan en los procesos transdisciplinarios de las ontologías

\begin{tabular}{|c|c|c|c|}
\hline Documentación & Normalización & & \\
\hline \multirow[t]{3}{*}{ Lingüística } & Lenguaje natural & & \\
\hline & PLN & Semántica formalizada & \\
\hline & Terminología & $\begin{array}{l}\text { Vocabularios } \\
\text { esquematizados }\end{array}$ & \\
\hline \multirow[t]{2}{*}{ Filosofia } & Lógica & Lógica matemática & $\begin{array}{l}\text { Schemas de } \\
\text { metadatos }\end{array}$ \\
\hline & Metafisica & Ontología & \\
\hline \multirow[t]{3}{*}{ Informática } & Inteligencia artificial & & \\
\hline & $\begin{array}{l}\text { Bases de } \\
\text { conocimientos }\end{array}$ & Modelos de datos & $\begin{array}{l}\text { Lenguajes de } \\
\text { marcado }\end{array}$ \\
\hline & $\begin{array}{l}\text { Desarrollo de } \\
\text { software }\end{array}$ & & \\
\hline Matemáticas & Algoritmos & & \\
\hline \multirow{3}{*}{$\begin{array}{l}\text { Teoría de } \\
\text { grafos }\end{array}$} & Educación & Mapas de conceptos & \\
\hline & Sociología & & \\
\hline & Psicología & & \\
\hline
\end{tabular}

Fuente: Elaboración propia

De las dos formas de mejorar la web, una sigue el camino tradicional de potenciar el procesamiento automático del lenguaje (PLN, por sus siglas en inglés) mediante el empleo de algoritmos de Inteligencia Artificial (IA) y de posicionamiento; por otro lado, la web semántica plantea nuevos sistemas conceptuales a partir de esquemas que mejoran la interoperabilidad y la legibilidad automáticas. Para conseguirlo, los documentos tienen que estar estructurados semánticamente y formalizados; con tal fin se precisa un buen desarrollo de software y normas incorporadas a esos documentos.

Esta es la base de las ontologías: semántica formalizada en schemas de metadatos codificados en un lenguaje de marcado, como XML, legible por 
ordenador; así, los conceptos son interpretables por las máquinas cuya jerarquía y asociaciones se hacen manifiestas mediante la lógica, lo que permite los procesos deductivos para realizar inferencias, y la validación de los axiomas y reglas de consistencia que posibilitan la conversión entre vocabularios. El modelo de datos lo proporciona RDF, mientras que el vocabulario para definir clases, propiedades, subclases, dominio, categorías, comentarios y etiquetas RDF — a ello se añade la consideración de las recomendaciones sobre confiabilidad (agentes, agencias de certificación...) - se atienen a un tiempo y a un espacio concretos, respondiendo, de esta manera, a la complejidad informativa y al contexto de las diferentes representaciones semánticas. Sin duda, ante situaciones de mayor complejidad había que dar soluciones conjuntas provenientes de múltiples dominios, actuando en coordinación e integración de procesos.

\section{Reflexión conclusiva}

La Bibliotecología y Documentación siempre ha contribuido a la comunicación científica. Además, ha facilitado el control y el acceso a la información en interactuación con todas las disciplinas científicas y humanas. Su nacimiento y consolidación se basaron en aportaciones multidisciplinarias. Asimismo, su práctica siempre se efectuó en contextos interdisciplinarios que acabarían siendo también la base de su justificación gnoseológica. De esta forma, desde los orígenes su existencia se enriqueció con la participación de diferentes conocimientos — rasgo multidisciplinario - y manifestó reciprocidad epistemológica y metodológica característica interdisciplinaria-.

La interdisciplinariedad es una exigencia para que nuestra especialidad pueda existir. Desde los orígenes históricos, nuestra naturaleza se razonó desde la contribución cooperativa con otros ámbitos cognitivos. Así, se acentuó cuando las tecnologías informáticas y de telecomunicaciones contribuyeron a mejorar el tratamiento de la información. A lo largo de la evolución científica, los métodos provenientes de otras disciplinas se han ido trasladando a nuestro dominio, en contribución ineludible, para fundamentar los continuos cambios y perfeccionamientos, tanto en los fundamentos teóricos como en los apoyos 
técnicos; ello dentro de la mejora continua de los métodos transferidos que pasan a modificarse o ampliarse. De esta manera, se llegan a presentar intensos cambios disciplinarios cuando fue imperioso alcanzar un nuevo paradigma, como sucedió al sobrevenir la Ciencia de la Información.

Ahora la resolución de los complicados problemas de la información ha hecho de la transdisciplinariedad el medio hacia una ciencia más completa cuando el diálogo de disciplinas integra los conocimientos de campos dispares en una visión de conjunto. La Bibliotecología y Documentación está comprometida en los procesos activos para el estudio de hechos en los que coinciden varios niveles de realidad. Ha permeabilizado aún más sus límites disciplinarios y se ha abierto hacia una teoría y práctica transdisciplinarias, en cuanto adaptación a las nuevas exigencias sociales y científicas de una sociedad compleja y muy distinta de la existente hace solo unas décadas, en especial en su relación con la información y la comunicación. Por eso, su base disciplinaria está en constante construcción y su aplicación en adaptación permanente a los cambios de las propias ciencias a las que auxilia, de las tecnologías que la apoyan y de las necesidades sociales a las que debe responder.

\section{Referencias bibliográficas}

Aristóteles. (1995). Tratados de Lógica: Organon, v. 1. Madrid: Gredos.

Bolaños, C. M., Bueno, G. F., Alves, F. M. (2013). El protagonismo de la Información-Documentación en la cooperación al desarrollo: los recursos educativos abiertos en los procesos de e-learning. Informação \& Sociedade, 23(1), 105-115.

BSI Group. (2005-2007). Structured vocabularies for information retrieval: guide. Londres: British Standards Institution.

Georgia Institute of Technology. (1962). Proceedings of the conferences on training Science Information Specialist. Atlanta: GIT.

International Organization for Standardization - ISO 2788 (1986). Documentation - Guidelines for the establishment and development of monolingual thesauri, segunda edición. Ginebra: ISO. 
International Organization for Standardization - ISO 25964-1. (2011-2013). Information and documentation - Thesauri and interoperability with other vocabularies. Ginebra: ISO.

Martínez, M. (2003). Transdisciplinariedad un enfoque para la complejidad del mundo actual. Conciencia activa, 21(1), 107-146.

Millares Carlo, A. (1971). Introducción a la historia del libro y de las bibliotecas. Ciudad de México: Fondo de Cultura Económica.

Mooers, C. (1959). Computer and Information Science Program. Filadelfia: Universidad de Pensilvania.

Moreno Fernández, L. M. \& Borgoñós Martínez, M. D. (1999). Teoría y práctica de la Clasificación Decimal Universal (CDU). Gijón: Trea.

Morin, E. (1990). Introducción al pensamiento complejo. París: ESPF Éditeur.

Newell, W. H. (2001). A theory of interdisciplinary studies. Issues in Integrative Studies, 19, 1-25. Recueprado de http://web.mit.edu/jrankin/www/ interdisciplinary/interdisc_Newell.pdf

Ortega y Gasset, J. (1984). La misión del bibliotecario. En El libro de las misiones, 10. ${ }^{\text {a }}$ edición. Madrid: Espasa-Calpe.

Otlet, P. (1934). Traité de Documentation. Le livre sur le livre. Théorie et pratique. Bruselas: Mundaneum.

Pérez Matos, N. E., y Setién Quesada, E. (2008). La interdisciplinariedad y la transdisciplinariedad en las ciencias: una mirada a la teoría bibliológicoinformativa. Acimed, 18(4). Recuperado de http://scielo.sld.cu/pdf/aci/ v18n4/aci31008.pdf

Sánchez Cuadrado, S., Colmenero-Ruiz, M. J. \& Moreiro-González, J. A. (2012). Tesauros: estándares y recomendaciones. El profesional de la información, 21(3), 229-235.

Saracevic, T. (1995). Interdisciplinary nature of information science. Ciência da informação, 24(1), 36-41. Recuperado de http://www.brapci.inf.br/ repositorio/2010/03/pdf_dd085d2c4b_0008887.pdf

Taylor, R. S. (1966). Professional aspects of information science and technology. Annual review of information science and technology, 1, 15-40. 
Taube, M. et. al. (1953-1959). Studies in coordinate indexing, 5 v. Washington: Documentation Inc.

Velilla, M. A. (2002). Manual de iniciación pedagógica al Pensamiento complejo. París: ICFES, UNESCO. Recuperado de http://online.upaep. $\mathrm{mx} /$ campusvirtual/ebooks/ManualIniciacion.pdf

Weinberg, A. (1963). Science, government, and information : the responsibilities of the technical community and the government in the transfer of information. [Washington, D. C.]: Government Printing Office. Recuperado de https:// files.eric.ed.gov/fulltext/ED048894.pdf

Wellisch, H. (1972). From Information Science to Informatics: a terminological investigation. Journal of Librarianship, 4(3), 157-187. doi: 10.1177/096100067200400302

Z3919:2005 (2005) ANSI/NISO. Guidelines for the Construction, Format, and Management of Monolingual Controlled Vocabularies. Bethesda, Maryland: NISO Press. 